\title{
Properties of the specific heat and chemical potentials of hadronic matter from CERN/RHIC experiments at relativistic and ultrarelativistic collision energies
}

\author{
Aram Mekjian \\ Rutgers University, Department of Physics, Piscataway, New Jersey 08854, USA \\ and \\ California Institute of Technology, Kellogg Radiation Laboratory Pasadena, California 91125, USA
}

(Received 25 February 2005; published 5 January 2006)

\begin{abstract}
The specific heat, mean hadronic mass excited and its fluctuation are connected to particle production yields and properties of baryon and electric charge chemical potentials (value, slope and curvature). A possible divergence of the specific heat as $1 /\left(T_{0}-T\right)^{2}$ is discussed. A Hagedorn model with $\rho \sim m^{-\tau} \exp \left(\beta_{h} m\right)$ is studied and restriction on $\tau$ are analyzed. Limitations imposed by a Q-g transition are mentioned.
\end{abstract}

DOI: 10.1103/PhysRevC.73.014901

PACS number(s): 25.75.Dw, 12.40.Ee, 21.65.+f, 25.75.Nq

\section{INTRODUCTION}

A statistical model of very high energy collisions can account for particle production yields from heavy ion collisions [1-5] and also in elementary particle collisions, such as electron-positron collisions [6,7]. The same model contains information regarding the thermodynamic properties of this system of particles. One important thermodynamic property is the specific heat. The importance of a study of the specific heat stems from the fact that sudden changes in the specific heat have been used as signals for phase transitions. A classic example of this statement is the lambda transition in liquid helium. The name lambda transition reflects the lambda shape of the specific heat with a very sharp rise followed by a sudden decrease. In the liquid-gas phase transition of nuclear matter at moderate excitation energy or temperature a very similar sharp peak in the specific heat was found in a theoretical model developed in Refs. [8,9]. For the situation discussed here, a rapid rise in the specific heat is associated with large fluctuations in the mass spectrum of excited particles. Event-by event studies [10] have also been stressed along with temperature fluctuations [11]. Large values of the specific heat are associated with large energy fluctuations. The compressibility is associated with density fluctuations [12]. Fluctuations associated with net electric charge and baryon charge have also been of recent interest $[13,14]$ as well as $p_{t}$ fluctuations [15]. An overview of fluctuations and correlations can be found in Refs. [16,17].

The organization of this paper is as follows. First, results of the statistical model are given for particle production yields and for thermodynamic quantities. The specific heat (here taken as the heat capacity per particle) is then connected to properties of the particle production yields and conserved charges such as baryon number $B$ and electric charge $Z$. Limiting cases of the specific heat are discussed which show a connection of it to the mass fluctuation in the spectrum of excited particles. Connections of the specific heat with the behavior of the chemical potential (its value, slope, and curvature) are also developed. The distribution of particles obtained from the detailed analysis of fitting the statistical model to hadronic multiplicities in $\mathrm{Pb}-\mathrm{Pb}$ collisions at $30 A, 40 A$ and $80 A \mathrm{GeV}$ data [3] is then used to study the behavior of the specific heat. A parametrization of the behavior of the chemical potential with $T$ from this analysis may indicate a sharp increase in the specific heat. A resonance gas model description of the behavior of the chemical potential with temperature is also given which has some similar properties.

\section{STATISTICAL MODEL}

\section{A. Statistical and thermodynamic properties}

The statistical model of heavy ion collisions assumes that hadron multiplicities are the result of an established thermal and chemical equilibrium [18] in some interaction volume $V$ at some temperature $T$. The interaction volume is the freeze-out volume which is the largest volume over which equilibrium is maintained in the evolution of the fireball. The underlying dynamical processes that establish the equilibrium are the collisions between the particles where the elastic collisions establish the thermal equilibrium and the inelastic collisions develop the chemical equilibrium. For example, pion production via a two-body collision is initially through a process $N+N \rightarrow N+N+\pi$, where $N$ is a nucleon. Since densities and energies are initially very high in the collision, three and higher order collision processes leading to two or more pions can also take place. Moreover, once new particles are produced through the initial collision of the nucleons in the colliding target and projectile nuclei in a nucleus-nucleus collision, these new particles can collide with existing nucleons or other newly created particles to produce other particles. As an example of a secondary process, kaons can be produced via pion induced reactions such as $\pi^{+}+\pi^{-} \rightarrow K^{+}+K^{-}$. A set of coupled reaction equations can be developed as in Ref. [18] to study the approach to equilibrium for each particle. The equilibrium thermodynamic model is based on the assumption that the reactions are initially fast enough compared to expansion time scales in the expanding fireball so that a quasiequilibrium is established. Since reaction rates depend on high powers of the density, i.e., the product of the densities of the colliding particles, 
the reaction rate decreases quickly and equilibrium cannot be maintained in an expanding system. A dynamical model of hadronization and freeze out can be found in Ref. [19]. The particle multiplicity distributions in an equilibrium model are given by

$$
\begin{aligned}
\left\langle N_{j}\right\rangle= & g_{j}\left(V m_{j}^{2} T / 2 \pi^{2}\right) \sum_{k}( \pm)^{k+1} K_{2}\left(k m_{j} / T\right) \\
& \times \operatorname{Exp}\left[b_{j} \mu_{B} k / T+q_{j} \mu_{Q} k / T\right] / k .
\end{aligned}
$$

The $b_{j}$ and $q_{j}$ are the baryon number and charge of particle $j$ which has spin degeneracy $g_{j}$ and mass $m_{j}$. The $\mu_{B}$ and $\mu_{Q}$ are the baryon and charge chemical potentials. The strangeness chemical potential $\mu_{S}$ will be set equal to 0 . The energy of particle $j$ is

$$
\begin{aligned}
\left\langle E_{j}\right\rangle= & g_{j}\left(V m_{j}^{3} T / 2 \pi^{2}\right) \sum_{k}( \pm)^{k+1}\left(\left(\frac{3}{4}\right) K_{3}+\left(\frac{1}{4}\right) K_{1}\right) \\
& \times \operatorname{Exp}\left(k\left(b_{j} \mu_{B}+q_{j} \mu_{Q}\right) / T\right) / k
\end{aligned}
$$

The arguments of the Bessel $K$ functions in Eq. (2) are the same as in Eq. (1). The sum over $k$ gives the degeneracy corrections, with the $k=1$ term the nondegenerate limit. The plus (+) sign is for bosons and the minus ( - ) sign is for fermions. For nonrelativistic particles in the nondegenerate limit,

$$
\left\langle N_{j}\right\rangle=g_{j}\left(V / \lambda_{j}^{3}\right) \operatorname{Exp}\left(\left(-m_{j}+b_{j} \mu_{B}+q_{j} \mu_{Q}\right) / T\right) .
$$

The $\lambda_{j}=h /\left(2 \pi m_{j} T\right)^{1 / 2}$. The $\left\langle E_{j}\right\rangle=\left\langle N_{j}\right\rangle\left(m_{j}+(3 / 2) T\right)$. For zero mass particles: $\left\langle N_{j}\right\rangle=g_{j}\left(V T^{3} / \pi^{2}\right) \operatorname{Exp}\left(b_{j} \mu_{B}+\right.$ $\left.\left.q_{j} \mu_{Q}\right) / T\right),\left\langle E_{j}\right\rangle=3 T\left\langle N_{j}\right\rangle$.

\section{B. Features of the chemical potentials $\mu_{B}$ and $\mu_{Q}$}

The two chemical potentials are determined by the constraint conditions on total baryon number $B$ and total charge $Q$ or $Z$. Namely, $B=\Sigma_{j} b_{j}\left\langle N_{j}\right\rangle$ and $Z=\Sigma_{j} q_{j}\left\langle N_{j}\right\rangle$. Moreover, the derivative of these chemical potentials with respect to $T$ (all partial derivatives are at constant $V$ in this paper as is the heat capacity $C_{V}$ ) can be obtained from the conditions $\partial B / \partial T=0$ and $\partial Z / \partial T=0$. For $\left\langle N_{j}\right\rangle$ given by Eq. (3), then

$$
\begin{aligned}
& (3 B / 2)+M_{B} / T+R_{b b} f_{B}+R_{b q} f_{Q}=0, \\
& (3 Z / 2)+M_{Q} / T+R_{b q} f_{B}+R_{q q} f_{Q}=0 .
\end{aligned}
$$

The $M_{B}=\Sigma_{j} m_{j} b_{j} N_{j}, M_{Q}=\Sigma_{j} m_{j} q_{j} N_{j}, R_{b b}=\Sigma_{j} b_{j}^{2} N_{j}$, $R_{q q}=\Sigma_{j} q_{j}^{2} N_{j}, R_{b q}=\Sigma_{j} b_{j} q_{j} N_{j}, f_{B}=\partial \mu_{B} / \partial T-\mu_{B} / T$, and $f_{Q}=\partial \mu_{Q} / \partial T-\mu_{Q} / T$. In these equations $N_{j}=\left\langle N_{j}\right\rangle$. The coupled equations for $f_{B}$ and $f_{Q}$ can be solved to give $f_{B}=\left(-\left(3 B / 2+M_{B} / T\right) R_{q q}+\left(3 Z / 2+M_{Q} / T\right) R_{b q}\right) / R, f_{Q}=$ $\left(-\left(3 Z / 2+M_{Q} / T\right) R_{b b}+\left(3 B / 2+M_{B} / T\right) R_{b q}\right) / R$. The $R=$ $R_{b b} R_{q q}-R_{b q}^{2}$. For massless pions the $f_{B}$ and $f_{Q}$ become $f_{B}=\left(-\left(3 B / 2+M_{B} / T\right) R_{q q}+\left(3 Z / 2+3 Z_{\pi} / 2+M_{Q} / T\right)\right.$ $\left.R_{b q}\right) / R$, and $f_{Q}=\left(-\left(3 Z / 2+3 Z_{\pi} / 2+M_{Q} / T\right) R_{b b}+(3 B / 2+\right.$
$\left.\left.M_{B} / T\right) R_{b q}\right) / R$. The $Z_{\pi}=N_{\pi^{+}}-N_{\pi^{-}}$, while the $Z$ is the total conserved charge.

\section{Expressions for the heat capacity and specific heat of hadronic matter}

The heat capacity $C_{V}$ in the nondegenerate and nonrelativistic limit for all particles is

$$
\begin{aligned}
C_{V}= & \left(\frac{3}{2}\right) \sum_{j} N_{j}+\sum_{j}\left(\frac{3}{2}+\frac{m_{j}}{T}\right)^{2} N_{j} \\
& +\left\{-\left(\frac{3 B}{2}+\frac{M_{B}}{T}\right)^{2} R_{q q}-\left(\frac{3 Z}{2}+\frac{M_{Q}}{T}\right)^{2} R_{b b}\right. \\
& \left.+2\left(\frac{3 B}{2}+\frac{M_{B}}{T}\right)\left(\frac{3 Z}{2}+\frac{M_{Q}}{T}\right) R_{b q}\right\} / R \\
= & \left(\frac{3}{2}\right) \sum_{j} N_{j}+\sum_{j}\left(\frac{3}{2}+\frac{m_{j}}{T}\right)^{2} N_{j}-R_{b b}\left(f_{B}\right)^{2} \\
& -R_{q q}\left(f_{Q}\right)^{2}-2 R_{b q} f_{B} f_{Q} .
\end{aligned}
$$

This is obtained from $C_{V}=(\partial E / \partial T)_{V}$ using Eqs. (2) and (3). The resulting equation will involve terms like $\partial \mu_{B} / \partial T$ which are evaluated using the constraint equations that lead to Eq. (4). Derivations of the fundamental equations are given in Appendix A of this paper.The first term (3/2) on the right-hand side of Eq. (5) is just the ideal gas specific heat at constant $V$ of each nonrelativistic particle, with both mesons and baryons contributing. The second term involves the mass spectrum of all particles produced. The curly bracket or third term in the first equality has three contributions and involves the three coefficients $R_{b b}, R_{q q}$, and $R_{b q}$. The second and the third term arise from the possibility that particle distributions change with $T$ or $\partial N_{j} / \partial T \neq 0$. The second term will be canceled by the third term for a system which has $\partial N_{j} / \partial T=0$, for all $j$. Specifically, consider a system of $N_{p}$ protons and $N_{n}$ neutrons, so that $N_{p}=Z$ and $N_{p}+N_{n}=B$. Then $R_{b b}=B, R_{q q}=Z$, and $R_{b q}=Z$. When these results are substituted into Eq. (5), along with the results $M_{B}=m_{N} B$ and $M_{Q}=m_{N} Z$, the second and the third term exactly cancel and $C_{V}=(3 / 2) B$, the ideal gas result. The last equality in Eq. (5) replaces the curly bracket term with the functions $-R_{b b}\left(f_{B}\right)^{2}-R_{q q}\left(f_{Q}\right)^{2}-2 R_{b q} f_{B} f_{Q}$ and connects $C_{V}$ to $\mu_{B}$ and $\mu_{Z}$.

When pions are taken in the zero mass and nondegenerate limit, the $C_{V}$ is modified to

$$
\begin{aligned}
C_{V}= & \left(\frac{3}{2}\right) \sum_{j} N_{j}+\sum_{j}\left(\frac{3}{2}+\frac{m_{j}}{T}\right)^{2} N_{j}+12\left(N_{\pi^{+}}+N_{\pi^{-}}+N_{\pi^{0}}\right) \\
& +\left\{-\left(\frac{3 B}{2}+\frac{M_{B}}{T}\right)^{2} R_{q q}-\left(\frac{3 Z}{2}+\frac{3 Z_{\pi}}{2}+\frac{M_{Q}}{T}\right)^{2} R_{b b}\right. \\
& \left.+2\left(\frac{3 B}{2}+\frac{M_{B}}{T}\right)\left(\frac{3 Z}{2}+\frac{3 Z_{\pi}}{2}+\frac{M_{Q}}{T}\right) R_{b q}\right\} / R .
\end{aligned}
$$

The first two sums over $j$ on the right-hand side of this equation exclude the pion contribution in their evaluation. The pion 
contribution is now contained in the following terms in that equation. The third term is the direct contribution of the pion as if it were independent of the charge conservation law and the curly bracket term arises from the chemical potentials and associated constraints. The independent pion contribution can also be calculated using the results of Eqs. (1) and (2). The exact expression for $C_{V, m} / N_{m}$ of an unconstrained meson or boson including statistical corrections reads

$$
\begin{aligned}
& C_{V, m} / N_{m} \\
&=(m / T)^{2}\left\{\sum_{k} K_{2}(k m / T)+3(T / m) K_{3}(k m / T) / k\right\} / \\
& \sum_{k} K_{2}(k m / T) / k
\end{aligned}
$$

The $m \rightarrow 0$ limit of this equation is $C_{V, m} / N_{m}=12 \varsigma(4) / \varsigma(3)$. If statistical corrections are neglected this limit would be 12 . The nondegenerate and large $m / T$ limit of Eq. (7) is $C_{V, m} / N_{m}=(3 / 2+m / T)^{2}+3 / 2$, the characteristic dependence of the first two terms in Eq. (6).

\section{Simplified model 1; only conserved baryon charge}

To gain some further insight into properties of $C_{V}$, a simplified situation of conserved baryon charge will be considered. Then all charge and neutral states of the same baryon will have equal yields. Mesons and baryons will also completely decouple. The specific heat $C_{V}$ will be a sum of independent contributions from mesons given by Eq. (7) and constrained baryons. The contribution of baryons to $C_{V, B}$ :

$$
\begin{aligned}
C_{V, B}= & \frac{3}{2} \sum_{j} N_{j, B}+\sum_{j}\left(\frac{3}{2}+\frac{m_{j}}{T}\right)^{2} N_{j, B} \\
& -\left(\frac{1}{R_{b b}}\right)\left(\frac{3 B}{2}+\frac{M_{B}}{T}\right)^{2} .
\end{aligned}
$$

The sums over $j$ are over both baryons and antibaryons. Antibaryons are usually a small fraction of the baryon number. If we allow only baryons with $b_{j}=1$, then $R_{b b}=B$, and

$$
C_{V, B}=\frac{3}{2} B+B \frac{1}{T^{2}}\left(\left\langle m_{B}^{2}\right\rangle-\left\langle m_{B}\right\rangle^{2}\right)=\frac{3}{2} B+B \frac{1}{T^{2}}\left(\delta m_{B}^{2}\right),
$$

where $\left\langle m_{B}\right\rangle=\Sigma_{j} m_{j} N_{j, B} / B$, and $\left\langle m_{B}^{2}\right\rangle=\Sigma_{j} m_{j}^{2} N_{j, B} / B$. The enhancement to $C_{V, B}=(3 / 2) B$ involves the mean square fluctuation in the resonance mass excitation. The mean mass $\left\langle m_{B}\right\rangle=-3 T / 2+\left(R_{b b} / B\right)\left(\mu_{B} / T-\partial \mu_{B} / \partial T\right)$. The condition $\partial^{2} B / \partial T^{2}=0$ can be used to obtain an expression for the mean square fluctuation and $C_{V, B} / B$ as

$$
\begin{aligned}
\left(\delta m_{B}\right)^{2} & =\left\langle m_{B}^{2}\right\rangle-\left\langle m_{B}\right\rangle^{2}=-3 T^{2} / 2-T^{3} \partial^{2} \mu_{B} / \partial T^{2}, \\
C_{V, B} / B & =3 / 2+\left(\delta m_{B}\right)^{2} / T^{2}=-T \partial^{2} \mu_{B} / \partial T^{2} .
\end{aligned}
$$

\section{E. Role of antibaryons}

The presence of antibaryons will modify the results given in Sec. II D. For collision energies $\leqslant 100 A \mathrm{GeV}$ antibaryons make up a few percent of $B$. From Ref. [3], the antiproton, proton ratio is $\sim 2 \%$ for the $80 A \mathrm{GeV} \mathrm{Pb}+\mathrm{Pb}$ collision.
This ratio rises to $\sim 5 \%$ for the $158 \mathrm{~A} \mathrm{GeV}$ collision. For a $\mu_{B} \sim 300 \mathrm{MeV}$ and $T \sim 150, \exp \left(-2 \mu_{B} / T\right)=\exp (-4.) \sim$ $2 \%$, which determines the antiparticle/particle ratio in the absence of $\mu_{Z}$. The presence of both antibaryons with fraction $y=N_{\bar{B}} /\left(N_{B}+N_{\bar{B}}\right)$ and baryons with fraction $x=$ $N_{B} /\left(N_{B}+N_{\bar{B}}\right)$ leads to a modified form for the baryonic (plus antibaryonic) $C_{V, B}$ :

$$
\begin{aligned}
C_{V, B} /\left(N_{B}+N_{\bar{B}}\right)= & 3 / 2+\left(x\left(\delta m_{B}\right)^{2}+y\left(\delta m_{\bar{B}}\right)^{2}\right) / T^{2} \\
& +x y\left(3+\left(\left\langle m_{B}\right\rangle+\left\langle m_{\bar{B}}\right\rangle\right) / T\right)^{2} \\
= & 3 / 2+\left(\delta m_{B}\right)^{2} / T^{2}+4 x y\left(3 / 2+\left\langle m_{B}\right\rangle / T\right)^{2} .
\end{aligned}
$$

The last equality arises from $\left\langle m_{B}\right\rangle=\left\langle m_{\bar{B}}\right\rangle$ and $\delta m_{B}=\delta m_{\bar{B}}$. The specific heat is also

$$
\begin{aligned}
C_{V, B} /\left(N_{B}+N_{\bar{B}}\right)= & \left(\left(N_{B}+N_{\bar{B}}\right) / B\right)\left(T \partial^{2} \mu_{B} / \partial T^{2}\right) \\
& +4\left(N_{B} N_{\bar{B}} / B^{2}\right) f_{B}^{2} \\
= & -\operatorname{coth}\left(\mu_{B} / T\right) T \partial^{2} \mu_{B} /(\partial T)^{2} \\
& +\operatorname{csch}^{2}\left(\mu_{B} / T\right)\left(\partial \mu_{B} / \partial T-\mu_{B} / T\right)^{2} \\
= & -\partial\left(\operatorname{coth}\left(\mu_{B} / T\right) T^{2}\left(\partial\left(\mu_{B} / T\right) / \partial T\right)\right) / \partial T .
\end{aligned}
$$

When $N_{\bar{B}} \rightarrow 0$, or $\mu_{B} / T$ very large, the rhs of Eqs. (11) and (12) reduces to Eq. (10). The specific heat now involves both the curvature and slope of the chemical potential and the value of the chemical potential itself. In the limit $x=y=1 / 2$ the $C_{V, B}$ of Eq. (12) becomes the unconstrained limit: $C_{V, B} /\left(N_{B}+N_{\bar{B}}\right)=3 / 2+\left(9 / 4+3\left\langle m_{B}\right\rangle / T+\right.$ $\left.\left\langle m_{B}^{2}\right\rangle / T^{2}\right)$. The ideal gas limit of $C_{V, B} / B=3 / 2$ is realized in the limit $x=1, y=0$ and $\delta m_{B}=0$. The $\left\langle m_{B}\right\rangle$ and $\left\langle m_{B}^{2}\right\rangle-$ $\left\langle m_{B}\right\rangle^{2}=\left(\delta m_{B}\right)^{2}$ are given by

$$
\begin{aligned}
\left\langle m_{B}\right\rangle / T= & -3 / 2-\operatorname{coth}\left(\mu_{B} / T\right)\left(\partial \mu_{B} / \partial T-\mu_{B} / T\right), \\
\left(\delta m_{B}\right)^{2} / T^{2}= & -3 / 2-\operatorname{coth}\left(\mu_{B} / T\right) T \partial^{2} \mu_{B} /(\partial T)^{2} \\
& +\operatorname{csch}^{2}\left(\mu_{B} / T\right)\left(\partial \mu_{B} / \partial T-\mu_{B} / T\right)^{2} .
\end{aligned}
$$

Thus, the values of $\mu_{B}$, its derivative and curvature also contain the information necessary to evaluate various quantities of interest regarding the mass excitation.

\section{F. Some features of the statistical model analysis of CERN/RHIC data}

The result of the previous sections can be used to evaluate some features of recent CERN/RHIC data. In Ref. [3], the $T$ dependence of $\mu_{B}$ is given as $T=.167-.153 \mu_{B}^{2}$. The $T$ and $\mu_{B}$ are in GeV. It should be stressed that the values of $\mu_{B}$ of Ref. [3] are on the freeze-out surface which has a volume dependence not included in the above expressions. Here, a more general form for the $T$ dependence of $\mu_{B}$ is used, namely $T=T_{0}-a\left(\mu_{B}\right)^{1 / \beta}$. In the next section a discussion of how such a form may arise is developed in a resonance gas model with $V$ constant. Here it is used as a simple form to study. Then substituting this result into Eqs. (13), (14) gives for $T \rightarrow T_{0}$

$$
\begin{aligned}
\left\langle m_{B}\right\rangle / T & \rightarrow \beta \cdot T_{0} /\left(T_{0}-T\right), \quad C_{V, B} /\left(N_{B}+N_{\bar{B}}\right) \propto\left(\delta m_{B}\right)^{2} / T^{2} \\
& \rightarrow \beta \cdot T_{0}^{2} /\left(T_{0}-T\right)^{2} .
\end{aligned}
$$


TABLE I. Values of $C_{V, B},\left\langle m_{B}\right\rangle$, and $\delta m_{B}$ in $\mathrm{MeV}$ excited in various collisions. Main analysis A is the first set of numbers in each row, main analysis $B$ is the second set.

\begin{tabular}{cccccrrrr}
\hline \hline Energy & $T$ & $\mu_{B}$ & $\left\langle m_{B}\right\rangle$ & $\delta m_{B}$ & $C_{V, B} / B$ & $\left\langle m_{B}\right\rangle$ & $\delta m_{B}$ & $C_{V, B} /\left(N_{B}+N_{\bar{B}}\right)$ \\
\hline \multirow{2}{*}{11.6} & 118.1 & 555 & 1061 & 277 & 7.0 & 1071 & 277 & 7.0 \\
& 119.1 & 578 & 1094 & 286 & 7.3 & 1077 & 267 & 7.3 \\
30 & 139.5 & 428.6 & 1295 & 593 & 19.6 & 1297 & 611 & 20.7 \\
& 140.3 & 428. & 1316 & 612 & 20.6 & 1313 & 633 & 21.9 \\
40 & 147.6 & 380.3 & 1513 & 853 & 34.9 & 1517 & 914 & 39.9 \\
& 145.5 & 375.4 & 1426 & 770 & 29.5 & 1444 & 812 & 32.8 \\
80 & 153.7 & 297.7 & 1771 & 1216 & 64.0 & 1856 & 1380 & 82.1 \\
& 151.7 & 288.9 & 1629 & 1070 & 51.2 & 1715 & 1187 & 62.7 \\
158 & 157.8 & 247.3 & 2113 & 1676 & 114.3 & 2331 & 2038 & 168.4 \\
& 154.8 & 244.5 & 1804 & 1313 & 73.4 & 1952 & 1513 & 97.0 \\
\hline \hline
\end{tabular}

Thus, $\beta$ does not appear as an exponent in the divergence of the specific heat which is quadratic or $1 /\left(T_{0}-T\right)^{2}$ for all $\beta$. The $\left\langle m_{B}\right\rangle \rightarrow \infty$ as $T \rightarrow T_{0}$. Using values for $\mu_{B}$ and $T$ given in Ref. [3] for $\mathrm{Pb}+\mathrm{Pb}$ collisions at $30,40,80$, and $158 \mathrm{~A} \mathrm{GeV}$ and for $\mathrm{Au}+\mathrm{Au} 11.6 A \mathrm{GeV}$ collisions, and the above parametrization of the behavior of $\mu_{B}$ with $T$ with $\beta=$ $1 / 2$, the values of $\left\langle m_{B}\right\rangle,\left(\left\langle m_{B}^{2}\right\rangle-\left\langle m_{B}\right\rangle^{2}\right)^{1 / 2} \equiv \delta m_{B}$ in $\mathrm{MeV}$ and $C_{V, B} / B$ are shown in Table I. The last three columns are obtained from results in Sec. II E that include antibaryons. The previous three columns are without antibaryons obtained from expressions in Sec. II D. The curvature and slope functions are obtained from this parametrization. The antibaryon case also used this parametrization to evaluate the chemical potential, while the case with just baryons used the chemical potential of Ref. [3]. The error bars in $T$ and $\mu_{B}$ are not given and generate large error bars in the results for the mean mass, mass fluctuation, and specific heat, especially at the higher temperatures. These errors are typically $\pm 20 \%$. Two sets of numbers for each energy appear in the table since Ref. [3] has two main analysis of the data, called $\mathrm{A}$ and $\mathrm{B}$. The results show that $C_{V, B} /\left(N_{B}+N_{\bar{B}}\right)$ are very different from the ideal gas value 1.5 and increase very sharply.

The contribution of decoupled mesons to $C_{V}$ can be obtained from Eq. (7). In Ref. [3]: $\pi^{\prime} s=1356, K^{\prime} s=160$, $K^{* \prime} s=66, \rho^{\prime} s=149, \eta=49, \omega=40$, are the multiplicity yields for the $80 \mathrm{~A} \mathrm{GeV} \mathrm{Pb}+\mathrm{Pb}$ collision for the lowlying well-known mesons. Using Eq. (7), each of these mesons contributes to $C_{V}$ as follows: $C_{V, \pi} / N_{\pi}=13.2, C_{V, K} /$ $N_{K}=28.8, C_{V, \eta} / N_{\eta}=32, C_{V, \omega} / N_{\omega}=49.5, C_{V, \rho} / N_{\rho}=48.5$, $C_{V, K^{*}} / N_{K^{*}}=59$. Thus mesons make a very large contribution to the total $C_{V}$.

\section{G. Resonance gas model}

The behavior of the chemical potential used in the previous section suggests a further investigation in terms of a Hagedorn resonance gas model. The Hagedorn model involves an exponentially increasing density of states that reads $\rho=$ $D_{\tau} m^{-\tau} \exp \left(\beta_{h} m\right)$, where $D_{\tau}$ is a constant and $\beta_{h}$ is the reciprocal of the limiting temperature $T_{0}$. For a system with net baryon number $\left(B=N_{B}-N_{\bar{B}}\right)$, the constraint imposed by baryon conservation leads to

$$
\begin{aligned}
& \left.\left(N_{B}-N_{\bar{B}}\right) / V\right)(2 \pi / T)^{3 / 2} \\
& \quad=2 D_{\tau}\left(y / m_{0}^{2}\right)^{\tau-5 / 2}\left(\int_{y}^{\infty}\left(d x e^{-x} / x^{(\tau-3 / 2)}\right)\right) \sinh \left(\mu_{B} / T\right)
\end{aligned}
$$

with $y=\left(T_{0}-T\right) m_{0} / T_{0} T$ and $m_{0}$ the lowest mass. For $\tau<5 / 2, \mu_{B}$ will go to zero as $\left(T_{0}-T\right)^{(5 / 2-\tau)}$, when $T \rightarrow T_{0}$. Thus, in the resonance gas model, the Hagedorn limiting temperature is identified with the limiting value of the chemical freeze-out temperature. The $I_{y, \tau} \equiv$ $\int_{y}^{\infty}\left(d x e^{-x} / x^{(\tau-3 / 2)}\right) \rightarrow \Gamma(5 / 2-\tau)$ for $y \rightarrow 0$. For $\tau>$ $5 / 2, \mu_{B}$ will go to a constant that depends on $\tau$. For $\tau=$ $5 / 2+\eta$, with $\eta>0, \quad(y)^{\tau-5 / 2} \int_{y}^{\infty} d x e^{-x} / x^{(\tau-3 / 2)}=1 / \eta$ as $y \rightarrow 0$. When $5 / 2<\tau<7 / 2$, the constant $1 / \eta$ is approached with $\infty$ slope. As an example $y^{1 / 2} I_{y, 3}=2\left(1-(\pi y)^{1 / 2}\right)$. For $7 / 2<\tau$, the slope is also finite: $y^{3 / 2} I_{y, 4}=(2 / 3)(1-2 y)$, $y^{2} I_{y, 9 / 2}=(1 / 2)(1-y)$. When $\mu_{B} \rightarrow\left(T_{0}-T\right)^{(5 / 2-\tau)}$ is substituted into Eq. (14), a divergence in $C_{V, B} /\left(N_{B}+N_{\bar{B}}\right)$ as $1 /\left(T_{0}-T\right)^{2}$ results. The $\left(N_{B}+N_{\bar{B}}\right) / V$ also diverges as $1 /\left(T_{0}-T\right)^{5 / 2-\tau}$, and $C_{V, B} \sim 1 /\left(T_{0}-T\right)^{9 / 2-\tau}$. The $N_{\bar{B}}, N_{B}$ asymmetry $\left(N_{B}-N_{\bar{B}}\right) /\left(N_{B}+N_{\bar{B}}\right)=\tanh \left(\mu_{B} / T\right)$, with $B=$ $N_{B}-N_{\bar{B}}$ fixed. This behavior of $C_{V, B}$ is the $B=0, \mu_{B}=0$ result of Ref. [20]. Earlier results at $\mu_{B} \neq 0, N_{\bar{B}}=0$ are in the second paper of Ref. [18]. A more recent discussion can be found in Ref. [21]. An $\infty$ baryon density presents a problem if baryons are not pointlike. The model is thus limited to densities and $T$ where composite baryons and mesons do not overlap. At some density $\sim 1$ hadron/ $/ \mathrm{fm}^{3}$ and $T=T_{Q g}<T_{0}$, a transition to a quark-gluon phase occurs which truncates behaviors based on this particular model. A discussion of this feature can be found in Ref. [21] where the quark-gluon phase is treated in a statistical model of quark-gluon bags along with the hadron phase.

How well does Eq. (15) describe the $\mu_{B}$ of Table I? When $\tau=2$, the $\mu_{B} \rightarrow\left(T_{0}-T\right)^{(1 / 2)}$ as $T \rightarrow T_{0}$ which is the form of Ref. [3] which will be used. Setting $\mu_{B}=375.5$ at $T=145.5$ and using $m_{0} / T_{0}=6 \sim m_{p} / T_{0}$ while also holding $V$ constant, gives $\mu_{B}=625,434,375,295$, and 250 at $T=119.1,140.3$, 145.5, 151.7, and 154.8, respectively. These values for $\mu_{B}$ 
can be compared to those in Table I which read for 578, $428,375,289,245$ for the same $T$ 's, respectively, which are on the freeze-out surface. The $V$ dependence of $\mu_{B}$ is contained in Eq. (15), but details of the freeze-out volume must be given. If $X$ is constant then $\left(\partial \mu_{B} / \partial T\right)_{X}-\left(\partial \mu_{B} / \partial T\right)_{V}=$ $\left(\partial \mu_{B} / \partial V\right)_{T}(\partial V / \partial T)_{X}$. In spite of this difference, Eq. (15) gives a good qualitative representation of $\mu_{B}$. The Hagedorn model has higher values of $C_{V, B} /\left(N_{B}+N_{\bar{B}}\right): 11.4,30.8,45.4$, 84.4 , and 129 , for these same $T$ 's.

\section{SUMMARY AND CONCLUSIONS}

Properties of the specific heat of hadronic matter produced in very high energy nucleus-nucleus collisions such as those at RHIC and CERN are studied in this paper. A grand canonical statistical model is used to develop expressions for the particle multiplicity distribution and energy caloric equation of state which is then used to obtain the specific heat. The constraints associated with baryon number and electric charge conservation are included to obtain an expression for the specific heat which contains the particle yields, the mass spectrum of produced particles, and three coefficients $R_{b b}, R_{q q}$, and $R_{b q}$ associated with these constraints. The specific heat is not simply a sum of independent contributions arising from each type of particle. Rather $C_{V}$ has additional terms which significantly alter its value from this independent particle result. Resonance excitations allow for the possibility that individual particle yields change with $T$ and redistribute the conserved charge and baryon number on other particles. The behavior of $C_{V}$ is studied in some limiting cases to see how various quantities such as baryonic charge conservation and the production of antibaryons manifest themselves. Using properties of the constraint equations, the specific heat and mass spectrum of excited hadrons are related to properties of $\mu_{B}$ and $\mu_{Q}$ such as their curvature, slope, and value. A recent parametrization of $\mu_{B}$ with $T$ as $\mu_{B}=a\left(T_{0}-T\right)^{1 / 2}$ may lead to a very rapid increase in the baryonic component of the specific heat. In general, if an expression such as $\mu_{B}=$ $a\left(T_{0}-T\right)^{\beta}$ correctly describes the behavior of $\mu_{B}$ near a limiting temperature $T_{0}$, then $C_{V, B} /\left(N_{B}+N_{\bar{B}}\right)$ would diverge as $1 /\left(T_{0}-T\right)^{2}$. Moreover, the exponent 2 is independent of the functional form of $\beta$ used. A resonance gas description of the behavior of the chemical potential and associated specific heat is also given. The mass prefactor exponent $\tau$ in a Hagedorn spectrum $\rho=D_{B} m^{-\tau} \exp \left(\beta_{h} m\right)$ is important in the behavior of the chemical potential. Specifically, if $\mu_{B} \rightarrow 0$, as $T \rightarrow T_{0}$, then $\tau \leqslant 5 / 2$, with $\mu_{B} \rightarrow\left(T-T_{0}\right)^{5 / 2-\tau}$. Thus, the Hagedorn limiting temperature of a resonance gas model can be identified with the limiting value of the chemical freeze-out temperature. Moreover, the behavior of $\mu_{B}$ may possibly be used to study $\tau$. The limitations of the Hagedorn result are discussed with respect to a $Q-g$ phase transition.

\section{ACKNOWLEDGMENT}

Research supported by DOE under Grant No. DOE: FG0296ER-40987.

\section{APPENDIX A: DERIVATION OF MAIN EQUATIONS}

This appendix contains details of the derivation of some of the equations used in this paper. To keep the notation simple, L > will be omitted and all partial derivatives with respect to $T$ are at constant $V$.

The heat capacity given by Eq. (4) is obtained from

$$
\begin{aligned}
C_{V} & =(\partial E / \partial T)_{V}=\frac{\partial}{\partial T} \sum_{j} N_{j}\left(m_{j}+\frac{3 T}{2}\right) \\
& =\frac{3}{2} \sum_{j} N_{j}+\sum_{j}\left(m_{j}+\frac{3 T}{2}\right) \frac{\partial N_{j}}{\partial T} .
\end{aligned}
$$

Using Eq. (3), the result for $\partial N_{j} / \partial T$ is

$$
\frac{\partial N_{j}}{\partial T}=\frac{N_{j}}{T}\left(\frac{3}{2}+\frac{m_{j}}{T}+b_{j} f_{B}+q_{j} f_{Q}\right),
$$

where $f_{B}=\partial \mu_{B} / \partial T-\mu_{B} / T$ and $f_{Q}=\partial \mu_{Q} / \partial T-\mu_{Q} / T$ arise from the partial derivatives of $\exp \left(\left(b_{j} \mu_{B}+q_{j} \mu_{Q}\right) / T\right)$ with respect to $T$. Substituting Eq. (A2) into Eq. (A1) leads to

$$
\begin{aligned}
C_{V}= & \frac{3}{2} \sum_{j} N_{j}+\sum_{j}\left(m_{j}+\frac{3 T}{2}\right) \\
& \times \frac{N_{j}}{T}\left(\frac{3}{2}+\frac{m_{j}}{T}+b_{j} f_{B}+q_{j} f_{Q}\right) \\
= & \frac{3}{2} \sum_{j} N_{j}+\sum_{j}\left(\frac{m_{j}}{T}+\frac{3}{2}\right)^{2} N_{j} \\
& +\sum_{j}\left(m_{j}+\frac{3 T}{2}\right) \frac{N_{j}}{T}\left(b_{j} f_{B}+q_{j} f_{Q}\right) \\
= & \frac{3}{2} \sum_{j} N_{j}+\sum_{j}\left(\frac{m_{j}}{T}+\frac{3}{2}\right)^{2} N_{j} \\
& +\left(\frac{M_{B}}{T}+\frac{3}{2} B\right) f_{B}+\left(\frac{M_{Z}}{T}+\frac{3}{2} Z\right) f_{Q} .
\end{aligned}
$$

When the results of Eq. (4) are substituted into Eq. (A3) the two forms of Eq. (5) in Sec. II B arise. Equation (8) of Sec. II D, which contains only the baryon constraint, is obtained in a similar manner. The baryonic component to the heat capacity is

$$
\begin{aligned}
C_{V, B}= & \frac{3}{2} \sum_{j} N_{j, B}+\sum_{j}\left(\frac{m_{j}}{T}+\frac{3}{2}\right)^{2} N_{j, B}+\left(\frac{M_{B}}{T}+\frac{3}{2} B\right) f_{B} \\
= & \frac{3}{2} \sum_{j} N_{j, B}+\sum_{j}\left(\frac{m_{j}}{T}+\frac{3}{2}\right)^{2} N_{j, B}+\left(\frac{M_{B}}{T}+\frac{3}{2} B\right) \\
& \times\left(-\frac{1}{R_{b b}}\right)\left(\frac{M_{B}}{T}+\frac{3}{2} B\right) .
\end{aligned}
$$

In the sums over $j$ in the last equation, the $N_{j, B}$ can either be a baryon or antibaryon. Both will contribute a plus sign to the baryonic specific heat. However, the $B$ and $M_{B}$ both involve the baryonic charge $b_{j}$ which has an opposite sign for baryons and antibaryons. In obtaining the last result of Eq. (A4), use 
was made of Eq. (4) for a situation with a constraint of baryon conservation only. Then Eq. (4) reads

$$
\frac{3}{2} B+\frac{M_{B}}{T}+R_{b b} f_{B}=0 .
$$

The $f_{B}$ of Eq. (A5) can be substituted into Eq. (A4) to eliminate its presence in that equation. The results of Eq. (A4) leads to Eq. (9) of Sec. II D (without antibaryons) using

$$
\begin{aligned}
& R_{b b}=B=N_{B}, \\
& M_{B}=\left\langle m_{B}\right\rangle B \\
& \sum_{j}\left(\frac{m_{j}}{T}+\frac{3}{2}\right)^{2} N_{j, B}=\left(\frac{\left\langle m_{B}^{2}\right\rangle}{T^{2}}+\frac{3\left\langle m_{B}\right\rangle}{T}+\frac{9}{4}\right) B .
\end{aligned}
$$

The results of Eq. (11) of Sec. II E (with antibaryons and $b_{j}= \pm 1$ only) also follow from Eq. (A4) using

$$
\begin{aligned}
R_{b b}= & \sum_{j} b_{j}^{2} N_{j, B}=N_{B}+N_{\bar{B}}, \\
B= & \sum_{j} b_{j} N_{j, B}=N_{B}-N_{\bar{B}}, \\
& \sum_{j} N_{j, B}=N_{B}+N_{\bar{B}}, \\
\left\langle m_{B}\right\rangle= & \left\langle m_{\bar{B}}\right\rangle, \quad\left\langle m_{B}^{2}\right\rangle=\left\langle m_{\bar{B}}^{2}\right\rangle, \\
M_{B} \equiv & \sum_{j} m_{j} b_{j} N_{j}=\left\langle m_{B}\right\rangle\left(N_{B}-N_{\bar{B}}\right)=\left\langle m_{B}\right\rangle B .
\end{aligned}
$$

Specifically

$$
\begin{aligned}
C_{V, B}= & \frac{3}{2}+\left(\frac{\left\langle m_{B}^{2}\right\rangle}{T^{2}}+3 \frac{\left\langle m_{B}\right\rangle}{T}+\frac{9}{4}\right)-\frac{B^{2}}{\left(N_{B}+N_{\bar{B}}\right)^{2}} \\
& \times\left(\frac{\left\langle m_{B}\right\rangle^{2}}{T^{2}}+3 \frac{\left\langle m_{B}\right\rangle}{T}+\frac{9}{4}\right) \\
= & \frac{3}{2}+\frac{\left(\partial m_{B}\right)^{2}}{T^{2}}+4 x y\left(\frac{3}{2}+\frac{\left\langle m_{B}\right\rangle}{T}\right)^{2},
\end{aligned}
$$

where

$$
\frac{B^{2}}{\left(N_{B}+N_{\bar{B}}\right)^{2}}=1-4 \frac{N_{B}}{N_{B}+N_{\bar{B}}} \frac{N_{\bar{B}}}{N_{B}+N_{\bar{B}}}=1-4 x y .
$$

The relationship that connects the mean mass excited to the chemical potentials follows from Eq. (4). For the case of baryon conservation only the first result in Eq. (4) with $f_{Q} \rightarrow 0$ can be written as

$$
\left(\frac{3}{2}+\frac{\left\langle m_{B}\right\rangle}{T}\right) B+\left(N_{B}+N_{\bar{B}}\right) f_{B}=0 .
$$

Thus

$$
\begin{aligned}
\frac{\left\langle m_{B}\right\rangle}{T} & =-\frac{3}{2}-\frac{\left(N_{B}+N_{\bar{B}}\right) f_{B}}{\left(N_{B}-N_{\bar{B}}\right)} \\
& =-\frac{3}{2}-\operatorname{coth}\left(\frac{\mu_{B}}{T}\right)\left(\frac{\partial \mu_{B}}{\partial T}-\frac{\mu_{B}}{T}\right) .
\end{aligned}
$$

The mean square fluctuation $\left(\partial m_{B}\right)^{2}$ is obtained from the constraint condition using $\partial^{2} B / \partial T^{2}=0=T\{(\partial / \partial T)$ $(T \partial B / \partial T)\} \equiv D_{2} B$ :

$$
\begin{aligned}
D_{2} B= & T \frac{\partial}{\partial T} \sum_{j} b_{j} N_{j}\left(\frac{3}{2}+\frac{m_{j}}{T}+b_{j} f_{B}\right) \\
= & \sum_{j} b_{j} N_{j}\left(-\frac{m_{j}}{T}+b_{j} T \frac{\partial f_{B}}{\partial T}\right) \\
& +\sum_{j} b_{j}\left(\frac{m_{j}}{T}+b_{j} f_{B}\right) T \frac{\partial N_{j}}{\partial T} .
\end{aligned}
$$

Using

$$
\begin{aligned}
T \frac{\partial f_{B}}{\partial T}= & T \frac{\partial^{2} \mu_{B}}{(\partial T)^{2}}-f_{B}, \\
& \frac{3}{2} B+\frac{\left\langle m_{B}\right\rangle}{T} B+R_{b b} f_{B}=0 \\
\frac{\left\langle m_{B}\right\rangle}{T} R_{b b} f_{B}= & \frac{\left\langle m_{B}\right\rangle}{T}\left(-\frac{3}{2} B-\frac{\left\langle m_{B}\right\rangle}{T} B\right),
\end{aligned}
$$

the $D_{2} B$ can be rewritten as

$$
\begin{aligned}
D_{2} B= & \frac{3}{2} B+R_{b b} T \frac{\partial^{2} \mu_{B}}{(\partial T)^{2}}+\frac{\left(\partial m_{B}\right)^{2}}{T^{2}} B \\
& -\left(\frac{\left(R_{b b}\right)^{2}}{B^{2}}-1\right) B\left(f_{B}\right)^{2}=0 .
\end{aligned}
$$

Solving for the mass fluctuation $\left(\partial m_{B}\right)^{2} / T^{2}$ gives

$$
\begin{aligned}
\frac{\left(\partial m_{B}\right)^{2}}{T^{2}}= & -\frac{3}{2}-\operatorname{coth}\left(\frac{\mu_{B}}{T}\right) T \frac{\partial^{2} \mu_{B}}{(\partial T)^{2}} \\
& +\csc h^{2}\left(\frac{\mu_{B}}{T}\right)\left(\frac{\partial \mu_{B}}{\partial T}-\frac{\mu_{B}}{T}\right)^{2} .
\end{aligned}
$$

The result of Eq. (A15) can be simplified to the following expression:

$$
-\frac{3}{2}-\frac{\partial}{\partial T}\left\{\operatorname{coth}\left(\mu_{B} / T\right)\left(T^{2} \frac{\partial\left(\mu_{B} / T\right)}{\partial T}\right)\right\} .
$$

[1] P. Braun-Munzinger and J. Stachel, Nucl. Phys. A606, 320 (1996).

[2] P. Braun-Munzinger, I. Heppe, and J. Stachel, Phys. Lett. B465, 15 (1999); P. Braun-Munziger et al., in Quark-Gluon Plasma 3, edited by R. C. Hwa and X.-N. Wang (World Scientific, Singapore, 2004).

[3] F. Becattini, M. Gazdzicki, A. Keranen, J. Mannienen, and R. Stock, Phys. Rev. C 69, 024905 (2004).

[4] J. Cleymans, H. Oeschler, and K. Redlich, Phys. Rev. C 59, 1663 (1999).
[5] J. Zimanyi, Nucl. Phys. A661, 224c (1999).

[6] F. Becattini, Z. Phys. C 69, 485 (1996).

[7] F. Becattini and U. Heinz, Z. Phys. C 76, 269 (1997).

[8] S. Das Gupta and A. Z. Mekjian, Phys. Rev. C 57, 1361 (1998).

[9] C. Das, S. Das Gupta, W. Lynch, A. Z. Mekjian, and B. Tsang, Phys. Rep. C 406, 1 (2005).

[10] E. V. Shuryak and M. A. Stephanov, Phys. Rev. C 63, 064903 (2001).

[11] L. Stodolsky, Phys. Rev. Lett. 75, 1044 (1995). 
[12] St. Mrowczynski, Phys. Lett. B430, 9 (1998).

[13] S. Jeon and V. Koch, Phys. Rev. Lett. 83, 5435 (1999); QuarkGluon Plasma 3 [2].

[14] M. Asakawa, U. Heinz, and B. Muller, Phys. Rev. Lett. 85, 2072 (2000).

[15] S. Gavin, Phys. Rev. Lett. 92, 162301 (2004).

[16] H. Heiselberg, Phys. Rep. 351, 161 (2001).
[17] J. T. Michell, J. Phys. G: Nucl. Part. Phys. 30, S819 (2004).

[18] A. Z. Mekjian, Phys. Rev. C 17, 1051 (1978); Nucl. Phys. A312, 491 (1978).

[19] L. Csernai and T. Csorgo, Phys. Lett. B333, 25 (1994).

[20] C. Hammer and S. Frautschi, Phys. Rev. D 4, 2125 (1971).

[21] M. Gorenstein, M. Gazdzicki, and W. Greiner, Phys. Rev. C 72 , 024909 (2005); arXiv:nucl-th/0505050. 